\title{
On the optimisation of the construction of a ground-based neutron monitor for galactic cosmic ray monitoring and space weather applications
}

\author{
Danislav Sapundjiev* \\ Royal Meteorological Institute of Belgium \\ Ringlaan 3 Avenue Circulaire \\ B-1180 Brussels \\ E-mail: danislav.sapundjiev@meteo.be
}

Stanimir M. Stankov

Royal Meteorological Institute of Belgium

Ringlaan 3 Avenue Circulaire

B-1180 Brussels

E-mail: S.stankovameteo.be

\section{Jean-Claude Jodogne}

Royal Meteorological Institute of Belgium

Ringlaan 3 Avenue Circulaire

B-1180 Brussels

E-mail: jcjod@meteo.be

The neutron monitor (NM) remains the best available instrument for monitoring the secondary nucleonic component of the galactic cosmic rays for more than 80 years. Recently, NMs have been given another role related to satellite-based technologies for monitoring and forecasting of space weather events. At many sites around the world, the old neutron monitors were being refurbished and their operation synchronised to form a network known as the neutron monitor data base (NMDB). During the years, little has been changed in the construction of the actual neutron monitor. In this work we carried out a detailed study of the role of the various components (reflector, producer and moderator) on the output signal of a NM64 neutron monitor. The study was performed using the fully integrated particle physics Monte Carlo simulation package FLUKA utilising several different types of incident particles - from monoenergetic neutrons and protons to a complex galactic cosmic rays source tailored for the location of the neutron monitor in Dourbes $\left(50.1^{\circ} \mathrm{N}, 4.6^{\circ} \mathrm{E}\right)$. The influence of the size and shape of the different parts on the energy spectrum of the neutrons in the detector tube was analysed in order to optimise the construction and to investigate if it would be possible to extract more information about the incident radiation. The results obtained here have been applied to the design of the second neutron monitor which is under construction in Dourbes.

The 34th International Cosmic Ray Conference,

30 July- 6 August, 2015

The Hague, The Netherlands

\footnotetext{
*Speaker.
} 


\section{Introduction}

The standard neutron monitor draws on the initial design of the "neutron monitor pile" introduced by Simpson et al.[1, 2] and the subsequent improvements carried out by Hatton and Carmichael [3] which led to the present most common type of neutron monitor used worldwide, the NM-64. Because of the rather large size of its detector tube $(17 \mathrm{~cm}$ in diameter and $210 \mathrm{~cm}$ in length) it was named "super neutron monitor". Over the past 50 years, the intensity of the Galactic Cosmic Rays (GCR) was regularly monitored at Dourbes $\left(50.1^{\circ} \mathrm{N}, 4.6^{\circ} \mathrm{E}\right)$ by nine NM-64 super monitors. In 2014 nine additional tubes, type LND 2061, were purchased in order to upgrade the existing monitor to a larger 18-tube monitor. A significant part of the upgrading process goes into investigating possible modifications in the original design of the monitor in order to improve the count rate and to develop applications for detection of solar particle events. In this paper we report on the results of our investigation on the effect of the neutron monitor components on the fluence of "slow neutrons" in the detector cavity where the detector tube is placed.

Previous efforts, mainly experimental, related to this subject have been reported by Carmichael et al. [4] who investigated different experimental configurations. More recently, Stoker et al. [5] discussed the issue by using computer codes with an attention to the response function of the neutron pile. Clem and Dorman [6] further elaborated on the response function of the NM64 using Monte-Carlo transport implemented by the FLUKA code[7].

In this work, we utilise the same transport code to investigate how the size of each neutron monitor component affects the flux of slow neutrons entering the detector region. In doing so, several simplifications were adopted. First, we consider the secondary neutrons at ground level to be the main contributor to the counting rate. Second, it is assumed that the detector tube is filled with Boron Trifluoride (BF-3) enriched in ${ }^{10} \mathrm{~B}$ (10-Boron). The results hold true for He-3 (Helium3 ) enriched detectors as well, since they are also sensitive to neutrons with energies up to about 1 $\mathrm{MeV}$. In other words, the evaluation of the monitor is done for secondary neutrons encountered in the detection area. Thus, the volume of the detecting tube becomes the only important parameter. In our case, it is the volume of the LND 2061 detection tube (diameter $152.4 \mathrm{~mm}$ and length 2087.8 $\mathrm{mm} /$ effective $1956.3 \mathrm{~mm}$ ).

\section{Specifications of the neutron monitor in Dourbes}

The cross-section of the Dourbes NM64 neutron monitor, as used in the calculations with FLUKA is shown in Fig.1. The outer region is the reflector which forms a rectangular box with thickness of $75 \mathrm{~mm}$. Inside the reflector is the lead producer (of rather complex shape) consisting of a lead ring of certain thickness and rectangular bodies (also made of lead) extending from its sides. Inside the producer there is a polyethylene cylinder with wall thickness of $10 \mathrm{~mm}$ called moderator. The LND 2061 counter tube is inserted inside the cylinder of the moderator.

\section{Source particles}

The first step during the simulation is the preparation of the input spectrum of source particles. Only the secondary spectrum of neutrons is considered here, obtained by FLUKA, and the transport of primary protons from the top of the atmosphere down to the Earth's surface. The simulation 


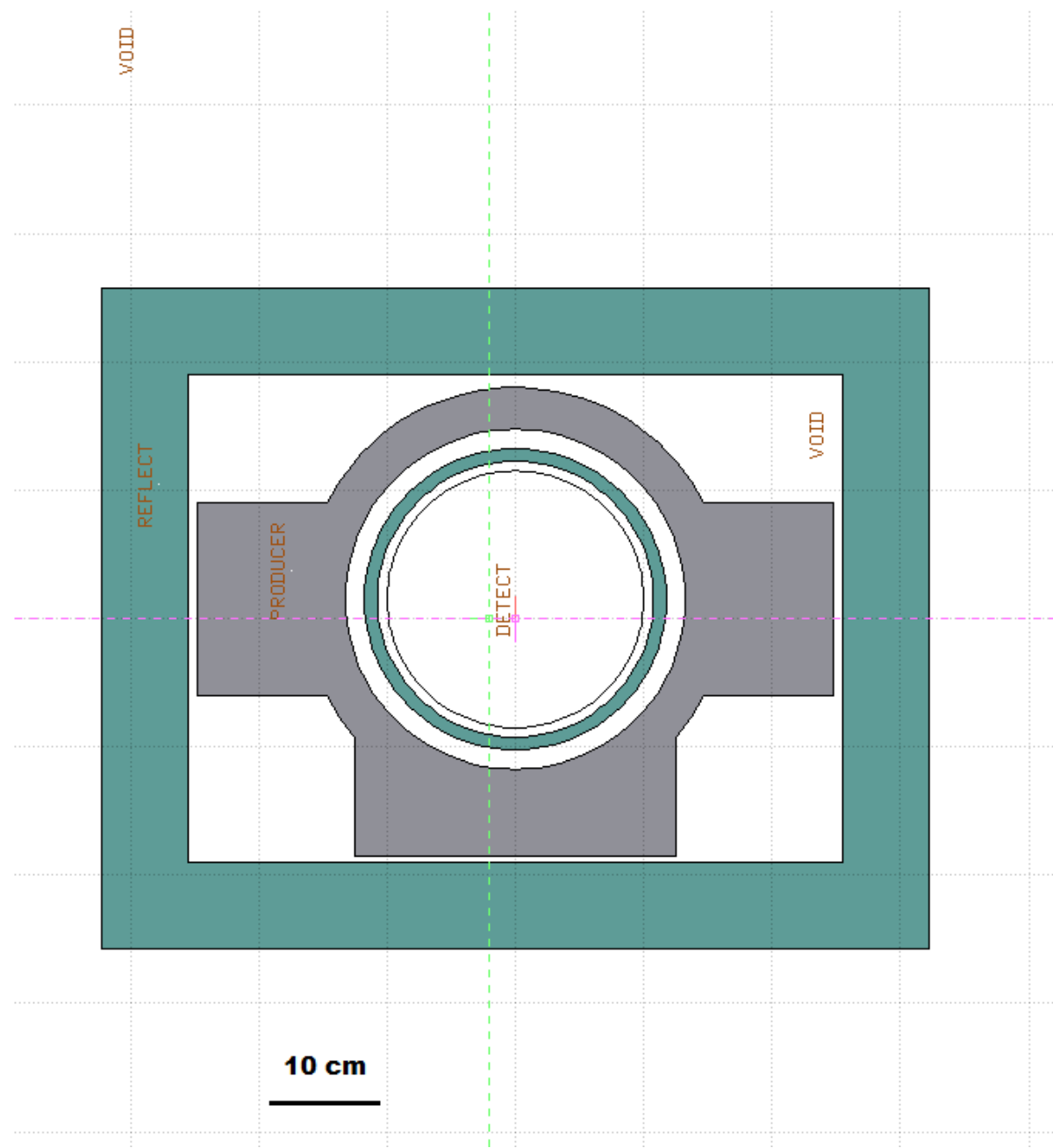

Figure 1: A schematic of a NM-64 single section: the cross section shows the different parts (reflector, producer, detector and moderator, the circle around the detector) considered for simulations with FLUKA.

is carried out for the geomagnetic cut-off and geographical position at Dourbes i.e. the simulation accounts for the trajectories of particles in the geomagnetic field and the transport through the atmosphere. In the simulation of the latter, 100 atmospheric layers are considered using the composition and density profiles of the US standard atmosphere. The GCR component was simulated using source particles with $\mathrm{Z}$ in the range of 1 to 28 and energies up to $30 \mathrm{TeV} /$ nucleon. Details about the primary particles spectrum, the atmospheric simulation, models, and how to simulate it in any particular case can be found in the FLUKA manual[7]. Note that, in our study, the modulation by the Sun is not considered. The resulting spectrum of secondary neutrons for Dourbes is plotted in Fig.2. This distribution was then used as an input for calculations with a slightly modified routine of FLUKA that samples the source particle energies accordingly. A requirement, stemming from the use of this distribution, is to have as little fluctuations as possible in order to decrease the spread in the ensuing simulation. This proved to be the most demanding (machine) calculation. 


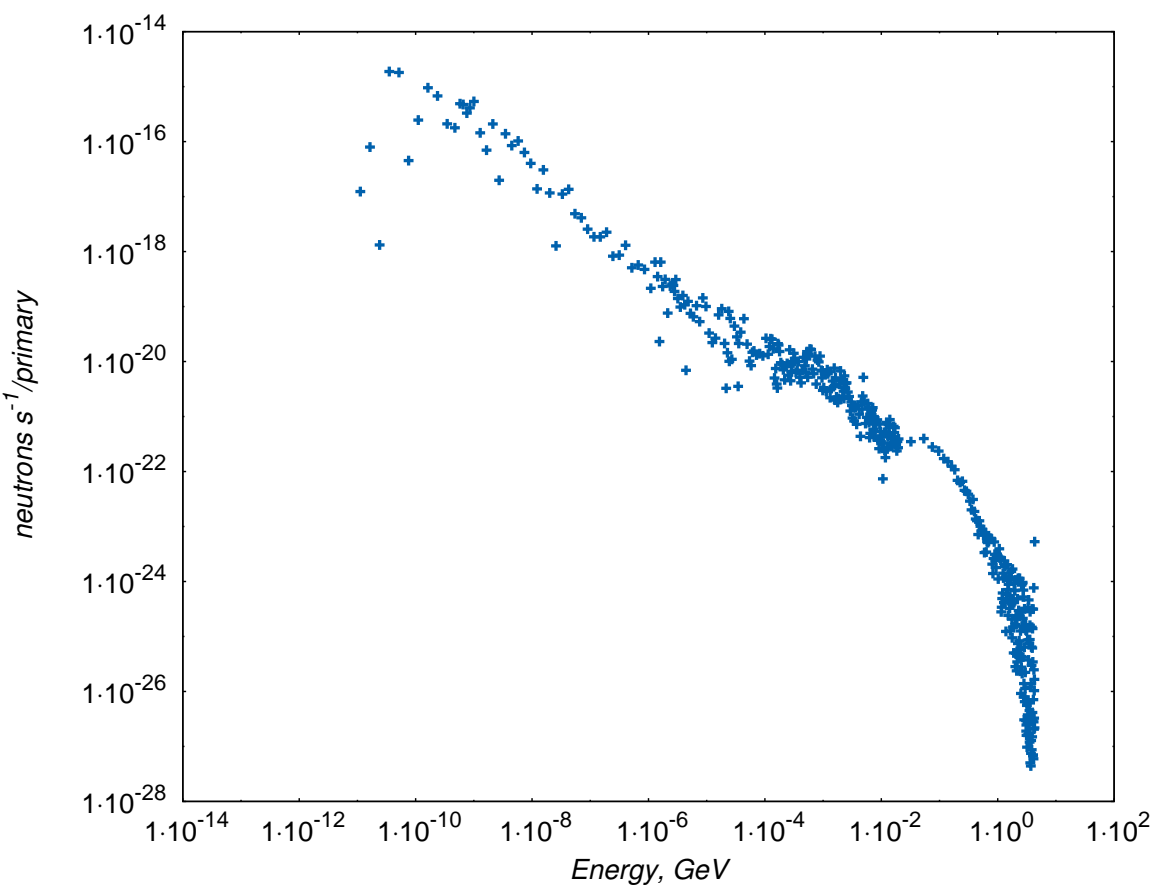

Figure 2: The simulated secondary neutron distribution for the site of Dourbes $\left(50.1^{\circ} \mathrm{N}, 4.6^{\circ} \mathrm{E}\right)$, based on simulation of $3 \times 10^{6}$ source particles.

\section{FLUKA optimisation of a NM64 monitor}

The actual optimisation was carried out by varying the dimensions of the different components of the neutron monitor, i.e. the moderator, the producer and the reflector. Since the size of the detector tube is fixed, in some cases there is little room for large variations (e.g. in the moderator). Therefore, the evaluation of the effects has been done by comparing of the fluence of neutrons (with energies of up to $1 \mathrm{MeV}$ ) of the original monitor and the same fluence calculated by changing the dimensions of its structural components. The test matrix is given in Table 1. For each run, 150000 source particles (with energies distributed according to Fig 2) were used.

\begin{tabular}{lcccccc}
\hline part & values, cm & & & & & \\
\hline moderator & 0 & 0.1 & 0.5 & $\mathbf{1}$ & 1.5 & 2 \\
producer & 0 & 2.25 & 2.75 & $\mathbf{3 . 2 5}$ & 3.75 & 4.35 \\
reflector & 0 & 2.5 & 5.0 & $\mathbf{7 . 5 5}$ & 10 & 12.5 \\
\hline
\end{tabular}

Table 1: Dimensions of the simulated components of the neutron monitor. The bold values are the dimensions of the existing monitor in Dourbes. The zero values indicate simulations without the corresponding part.

\subsection{Effect of the moderator thickness}

The resulting low energy neutron fluence $(<2 \mathrm{MeV})$ is plotted in Fig.3. It is clearly seen that the moderator significantly increases the number of neutrons with energies below $100 \mathrm{keV}$. 
Logically, the use of a thicker moderator should slow down neutrons with lower energy. The results show that the use of a thicker moderator leads to more neutrons with energy below $7 \mathrm{eV}$ but less neutrons with energy in the region $7 \mathrm{eV}-100 \mathrm{keV}$. The total fluence of neutrons in the low energy region (i.e. below $1 \mathrm{MeV}$ ) is given in Table 2 .

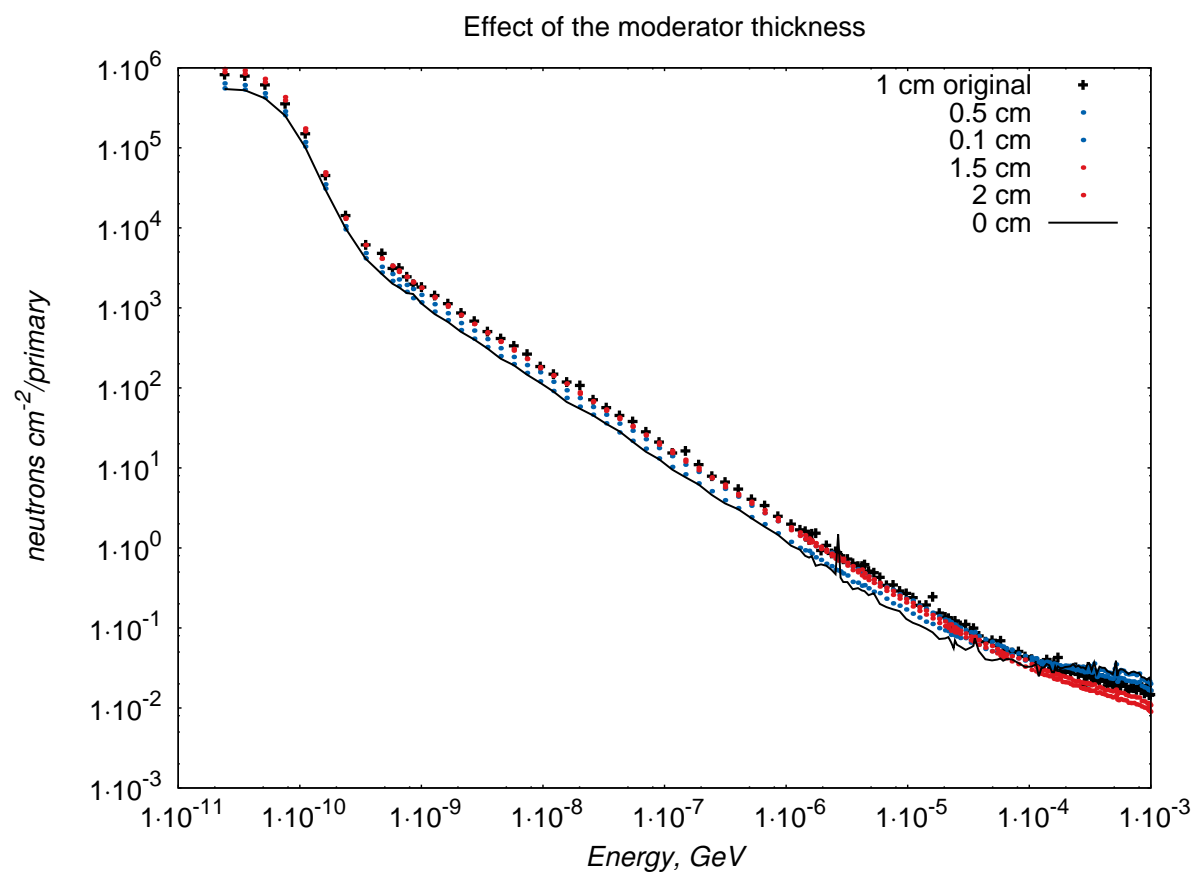

Figure 3: Low energy neutron fluence $(<1 \mathrm{MeV})$ encountered in the detector tube at different thicknesses of the moderator: zero thickness, i.e. no moderator (solid line), original thickness (crosses); greater thickness (red dots), lesser thickness (blue dots).

\begin{tabular}{lcccccc}
\hline thickness, cm: & 0 & 0.1 & 0.5 & $\mathbf{1}$ & 1.5 & 2 \\
\hline neutron fluence, $\times 10^{6} \mathrm{n} . \mathrm{cm}^{-2}$ & 1.88 & 1.89 & 2.22 & $\mathbf{2 . 8 2}$ & 3.10 & 3.31 \\
\hline
\end{tabular}

Table 2: Total low energy neutron fluence $(<1 \mathrm{MeV})$ without moderator, original moderator, and for moderators with different thicknesses. The fluence value/s at the original moderator thickness (10 $\mathrm{mm})$ given in bold numbers.

\subsection{Effect of producer thickness}

The simulations of the producer involved variations in the oval, ring-shaped part of the producer. The calculated low energy neutron spectrum in the region of the detector tube is plotted in Fig 4 together with the results for the original dimensions and for the case without lead producer. The presence of a producer significantly affects the number of low energy neutrons; however, the shape of the distribution remains the same. Variations in the dimensions of the producer ring show that the maximum fluence of neutrons below the $1 \mathrm{MeV}$ limit is achieved at the current size of the producer (cf. Table 3). Most probably, a larger producer would require a thicker moderator as well in order to slow down the neutrons produced in the lead to the energies of interest. This 
becomes obvious when looking at the fluence of neutrons at different dimensions of the producer for energies greater than $1 \mathrm{MeV}$ (cf. Table 3).

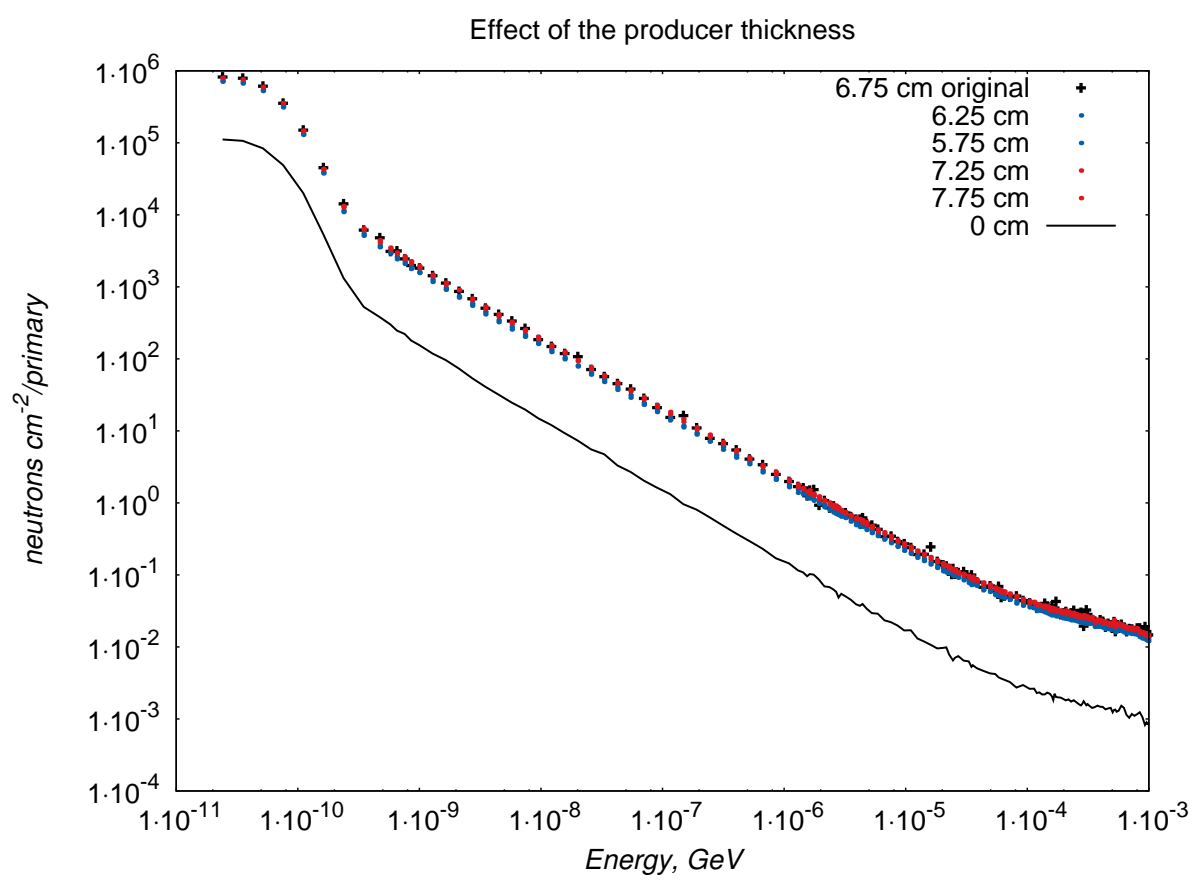

Figure 4: Effect of producer thickness - neutron fluence encountered in the detector tube for different thicknesses of the producer: zero thickness, i.e. no producer (solid line), original thickness (crosses); greater thickness (red dots), lesser thickness (blue dots).

\begin{tabular}{lcccccc}
\hline thickness, cm: & 0 & 5.75 & 6.25 & $\mathbf{6 . 7 5}$ & 7.25 & 7.75 \\
\hline neutron fluence $<1 \mathrm{Mev}, \times 10^{6} \mathrm{n} . \mathrm{cm}^{-2}$ & 0.39 & 2.41 & 2.52 & $\mathbf{2 . 8 2}$ & 2.73 & 2.78 \\
neutron fluence $[1 \mathrm{MeV}: 2 \mathrm{GeV}], \times 10^{-} 2{\mathrm{n} . \mathrm{s}^{-2}}^{-}$ & 0.04 & 0.29 & 0.29 & $\mathbf{0 . 3 3}$ & 0.32 & 0.33 \\
\hline
\end{tabular}

Table 3: Neutron fluence without producer, original producer, and for producer with different thicknesses. The fluence value/s at the original producer thickness $(32.5 \mathrm{~mm})$ given in bold numbers.

In the energy interval of $1 \mathrm{MeV}$ to $2 \mathrm{GeV}$ the statistics is low (i.e. the number of events registered during the simulation is very small) and the calculated values vary within the uncertainty of the results, the only exception being the calculation without producer. This indicates that the majority of neutrons evaporated from the producer are well limited to energies of $1 \mathrm{MeV}$ possibly because the faster ones undergo reflection and moderation from the moderator and/or the reflector. This results in multiplication of the produced neutrons with energies below $1 \mathrm{MeV}$ by a factor of 10. Although such a large factor suggests that it is worth investing into the material, the producer itself is not of such importance for the operation of the neutron monitor.

\subsection{Effect of reflector thickness}

The reflector fulfills a dual purpose: on the one hand, it has to prevent background neutrons 
of entering the monitor and, on the other hand, it has to reflect and moderate neutrons produced in the producer back towards the detector. In doing so, it must permit low-energy secondary neutrons, produced in the atmosphere from the primary cosmic rays, to reach the core (i.e. the detector) of the monitor. Additionally, it serves as a moderator for the secondary cosmic ray neutrons as well. The reflector is perhaps the most important (albeit the most difficult) part of a neutron monitor in terms of optimisation. This is confirmed by the results from the simulations (cf. Fig.5 and Table 4): the fluence of neutrons in the core of the monitor increases with the increase in reflector thickness until a certain maximum level is reached. Further increase in the reflector thickness results in decrease of the low energy neutron fluence.

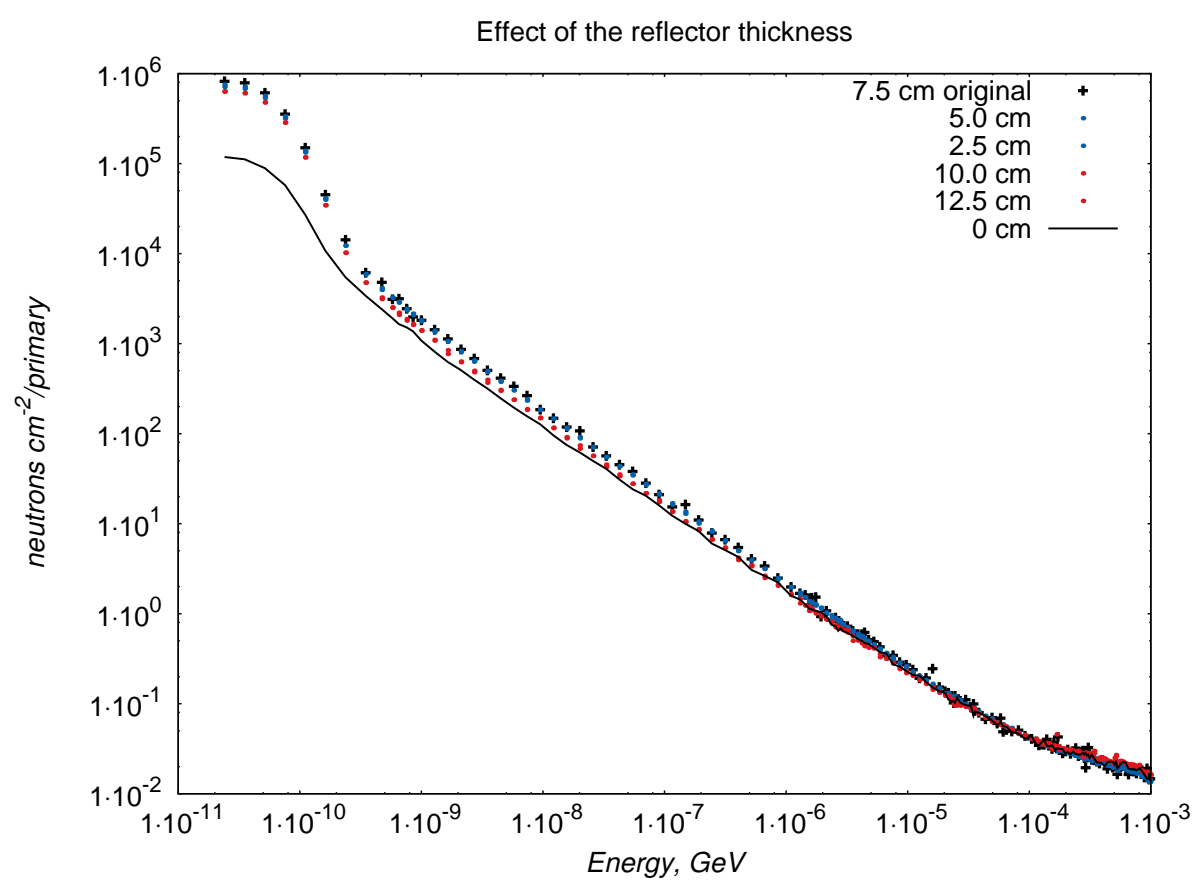

Figure 5: Effect of reflector thickness - neutron fluence encountered in the detector tube for different thicknesses of the reflector: zero thickness, i.e. no reflector (solid line), original thickness (crosses); greater thickness (red dots), lesser thickness (blue dots).

The moderating role of the reflector can be seen from the fluence calculated without the reflector: the fluence of neutrons with energies below $1 \mathrm{keV}$ is greatly reduced. These neutrons come from moderation of secondary atmospheric neutrons and from neutrons produced in the monitor and reflected (and/or moderated) in the reflector. Because in this study we do not consider any medium surrounding the monitor, we cannot completely comprehend the role of the reflector.

\begin{tabular}{lcccccc}
\hline thickness, cm: & 0 & 2.5 & 5 & $\mathbf{7 . 5}$ & 10 & 12.5 \\
\hline neutron fluence, $\times 10^{6} \mathrm{n} . \mathrm{cm}^{-2}$ & 0.46 & 2.40 & 2.55 & $\mathbf{2 . 8 2}$ & 2.20 & 2.09 \\
\hline
\end{tabular}

Table 4: Neutron fluence without reflector, original reflector, and for reflector with different thicknesses. The fluence value/s at the original reflector thickness $(75.5 \mathrm{~mm})$ given in bold numbers. 


\section{The optimised Neutron Monitor}

With the observed results, a slight modification of the existing NM64 neutron monitor in Dourbes was made and the resulting spectrum of low energy neutrons inside the detector has been calculated. In Fig 6 the spectrum is plotted for the original and the optimised monitor according to our calculations. The optimisation consisted in increasing the thickness of the moderator to 20 $\mathrm{mm}$ and the producer to $37.5 \mathrm{~mm}$ (the ring shaped region of the producer). The resulting total neutron fluence in the region of the detector increase from 2.82 to $2.94 \times 10^{6} \mathrm{n} . \mathrm{cm}^{-2}$, or about 1.05 times. This was achieved only with a simple optimisation scheme i.e. by varying one parameter (dimension) at a time and keeping the remaining parameters at their original values.

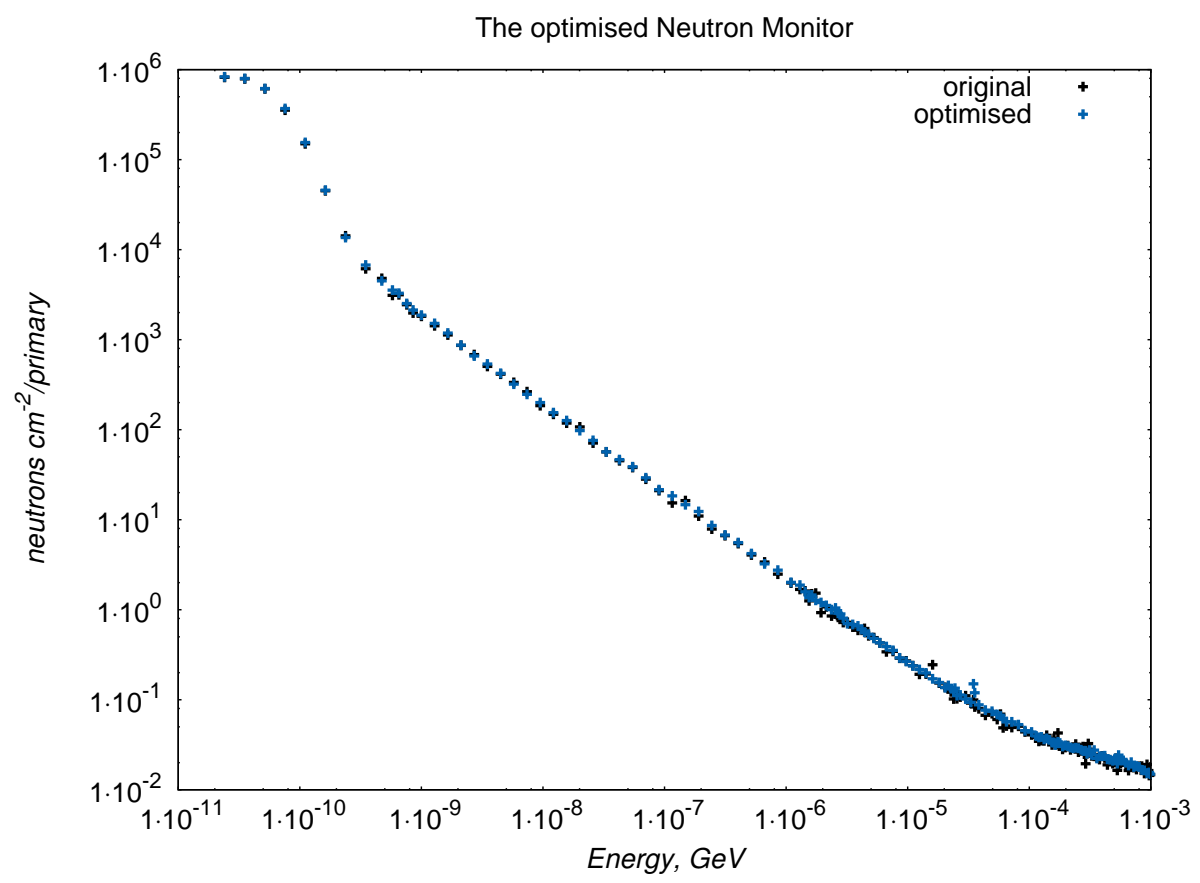

Figure 6: Comparison between the optimised and the original NM64 neutron monitor at Dourbes.

Additionally, in this preliminary study, only the fluence of neutrons in the detector region was considered; no background neutrons or other particles / photons were taken into account. The follow-on study would consider all possible factors that might affect the resulting counting rate of the NM64. In addition, the effect of the surroundings has to be studied: surroundings able to reflect neutrons are expected to contribute to the counting rate. Structural materials in the vicinity of the detector, that are capable of producing neutrons, can also be important for the total counting rate.

\section{Conclusions and outlook}

A preliminary optimisation study was conducted in order to maximise the neutron fluence in the detector region of a standard neutron monitor NM64. By varying the thickness of the moderator and producer, the resulting fluence of neutrons with energies below $1 \mathrm{MeV}$ was increased by 1.05. These results are encouraging and show that there is a room for improvement of the original 
neutron monitor still employed in Dourbes. However, the complete optimisation requires considering a number of additional factors and structural components among which the filler gas inside the detection tube, the medium surrounding the detector and additional components (particles and photons) in the secondary GCR spectrum at ground level. These factors will be investigated in a follow-on study and the feasibility of implementing changes in the monitor design will be thoroughly evaluated before the actual construction of the second monitor in Dourbes.

\section{References}

[1] J.A.Simpson, W.H.Fonger, and S.B.Treiman, Cosmic radiation intensity-time variations and their origin: I. neutron intensity variation method and meteorological factors, Phys. Rev. 90 (1953), no. 5 934-950.

[2] J.A.Simpson, The cosmic ray nucleonic component: The invention and scientific uses of the neutron monitor, Space Science Reviews 93 (2000), no. 1-2 11-32.

[3] C. J. Hatton and H. Carimichael, Experimental investigation of the nm-64 neutron monitor, Canadian Journal of Physics 42 (1964), no. 12 2443-2472, [http: / /dx.doi.org/10.1139/p64-222].

[4] H. Carmichael, M. Bercovitch, M. A. Shea, M. Magidin, and R. W. Peterson, Attenuation of neutron monitor radiation in the atmosphere, Canadian Journal of Physics 46 (1968), no. 10 S1006-S1013, [http://dx.doi.org/10.1139/p68-405].

[5] P. H. Stoker, L. I. Dorman, and J. M. Clem, Neutron monitor design improvements, Space Science Reviews 93 (2000), no. 1-2 361-380.

[6] J. Clem and L. Dorman, Neutron monitor response functions, Space Science Reviews 93 (2000), no. 1-2 335-359.

[7] A. Ferrari, P. Sala, A. Fassò, and J. Ranft, FLUKA: a multi-particle transport code, Tech. Rep. INFN/TC_05/11, SLAC-R-773, CERN, 10, 2005. 